\title{
ANDES

www.scielo.cl

\section{Crecimiento a 24 meses de prematuros menores de 32 semanas, con o sin restricción de crecimiento intrauterino o postnatal}

\author{
Growth until 24 months in preterm of very low birth weight, with or without \\ intrauterine or postnatal growth restriction
}

\section{Patricia Mena Nanniga ${ }^{\mathrm{a}}$ Patricia Vernal Silva ${ }^{\mathrm{b}}$, Alexis Díaz Gonzalez ${ }^{\mathfrak{c}}$, María Teresa Henríquez Höfter ${ }^{\mathrm{d}}$, Enrica Pittaluga Pierdiluca ${ }^{\mathrm{a}}$, Ivonne D’Apremont Ormeño ${ }^{\mathrm{a}}$, Mónica Morgues Nudman ${ }^{\mathrm{b}}$, Jane Standen Herliz ${ }^{\mathrm{e}}$, Valeria de Toro Navarrete ${ }^{\mathrm{f}}$}

\author{
aServicio de Neonatología, Hospital Sotero del Río. Santiago, Chile \\ bervicio de Neonatología, Hospital San José. Santiago, Chile \\ 'Servicio de Neonatología, Hospital Regional Libertador Bernardo O'Higgins. Rancagua, Chile \\ ¿Servicio de Neonatología Hospital San Juan de Dios. Santiago, Chile \\ eServicio de Neonatología, Hospital Dr. Gustavo Fricke. Viña del Mar, Chile \\ fPontificia Universidad Católica de Chile. Santiago, Chile
}

Recibido: 24 de marzo de 2021; Aceptado: 22 de junio de 2021

\section{¿Qué se sabe del tema que trata este estudio?}

El crecimiento de prematuros puede disminuir intrauterina, postnatal o postalta. Caracterizar el crecimiento posterior según estas alteraciones se ha descrito con retardo de crecimiento intrauterino, postnatal o ambos.

\section{¿Qué aporta este estudio a lo ya conocido?}

Se describe el crecimiento de prematuros sin alteración de éste, de prematuros con alteración de crecimiento postnatal, pero rápida recuperación postalta que continúan con buen crecimiento posterior, prematuros con restricción postnatal, con recuperación en los primeros 6 meses y prematuros con restricción intrauterino y postnatal que recuperan muy lentamente hasta los 2 años.

\section{Resumen}

El crecimiento de recién nacidos prematuros puede afectarse durante período fetal, la hospitalización y post alta. Objetivo: describir el crecimiento de prematuros, con o sin restricción de crecimiento intrauterino y postnatal, y con o sin recuperación a las 40 semanas desde el nacimiento hasta los 24 meses de vida. Pacientes y Método: Estudio descriptivo retrospectivo de crecimiento en puntaje Z (según Fenton y OMS) de peso, longitud, perímetro craneano y peso/talla de prematuros $<32$ semanas al nacer, hasta los 2 años de edad corregida. Se definieron cuatro grupos según crecimiento prenatal, postnatal y postalta. Grupo AAA: lactantes nacidos AEG, sin restricción postnatal; Grupo APA: lactantes nacidos AEG con restricción postnatal, con peso al alta $<\mathrm{p} 10$, y a las 40 semanas con
Palabras clave:

Restricción de

Crecimiento

Intrauterina;

Crecimiento Postnatal;

Muy Prematuros;

Puntaje Z;

Crecimiento

Recuperacional;

Relación Peso/Talla 
peso > p10; Grupo APP: lactantes nacidos AEG, con restricción postnatal, con peso al alta $<$ p10, que se mantienen bajo percentil 10 a las 40 semanas, y Grupo PPP: lactantes nacidos con restricción intrauterina y que mantuvieron restricción postnatal (al nacer, al alta y 40 semanas con peso < p10). Se utilizó estadística descriptiva, ANOVA y Chi cuadrado, y análisis de Modelos Lineales Mixtos. Resultados: Se incluyeron 721 prematuros, peso de 1272 g. (DE 360) y edad gestacional 29 (DE 1,9) semanas al nacer. El grupo AAA mantiene puntaje $Z$ de peso, longitud y perímetro craneano alrededor de la mediana, hasta los 2 años. Los prematuros adecuados al nacer y restricción postnatal, pueden tener dos trayectorias: un grupo presenta recuperación a las 40 semanas (Grupo APA) y otro grupo presenta puntaje $Z$ peso $<-1$ hasta los 6 meses (Grupo APP). El grupo PPP (con restricción intrauterina y postnatal) presenta lenta recuperación de puntaje $Z$ de peso y longitud con $Z$ de peso alta de $-2,3$ y lenta mejoría hasta $<-1$ a los 2 años. Todos los grupos presentaron $Z$ peso/talla sobre la mediana en los primeros 2 meses corregidos. Conclusión: Los prematuros con buen crecimiento intrauterino en peso, pero deterioro postnatal, pueden recuperarse a las 40 semanas, con evolución normal posterior o recuperarse a los 6 meses. El grupo de prematuros con restricción de crecimiento pre y post natal (PPP) mantiene el compromiso nutricional en los 2 primeros años de vida.

\section{Abstract}

The growth of preterm newborns can be affected during the fetal period, hospitalization, and postdischarge. Objective: to describe the anthropometric development of preterm newborns with or without intrauterine and postnatal growth restriction, and with or without recovery at 40 weeks from birth to 24 months of age. Patients and Method: Retrospective, descriptive study with Z-scores (Fenton and WHO) of weight, length, head circumference, and weight/length of preterm infants of less than 32 weeks of gestational age at birth up to 24 months of corrected age. 4 groups were defined according to prenatal, postnatal, post-discharge growth as follows: Group AAA: newborns born AGA, with no postnatal growth restriction; Group APA: newborns born AGA, with postnatal growth restriction, weight $<$ p10 at discharge, and weight $>$ p10 at 40 weeks; Group APP: newborns born AGA, with postnatal growth restriction, weight $<$ p10 at discharge and at 40w; and Group PPP: newborns born with intrauterine growth restriction and who maintained postnatal growth restriction $(<\mathrm{p} 10$ at birth, at discharge, and at 40w). We used descriptive statistics with ANOVA, Chi-squared, and linear mixed model analysis. Results: 710 preterm newborns were included, birth weight 1272 grams (SD 360 ) and gestational age 29 weeks (SD 1.9). Group AAA had weight, length, and head circumference Z-scores close to the median until 2 years of age. AGA preterm newborns and with postnatal growth restriction can evolve in two ways: one group presents recovery at 40 weeks (Group APA) while the other group presents weight Z-score $<-1$ up to 6 months (Group APP). Group PPP (with intrauterine and postnatal growth restriction) presents slow weight and length Z-score recovery, weight Zscore -2.3 at discharge, and slow improvement to $<-1$ at 2 years of age. All groups had weight/height $\mathrm{Z}$-scores above the median in the first 2 months of corrected age. Conclusion: Preterm newborns with good fetal growth but restricted postnatal growth, may recover at 40 weeks, with subsequent normal development or recover at 6 months.

\section{Keywords:}

Fetal Growth

Restriction;

Postnatal Growth;

Compensatory Growth;

Extremely Preterm

Infant;

Z-Score;

Weight/Length Ratio

\section{Introducción}

La mortalidad neonatal del prematuro se ha reducido en las últimas décadas, logrando la sobrevida de niños de menor peso al nacer ${ }^{1}$. Uno de los problemas relevantes y aún pendiente, es alcanzar un óptimo crecimiento en prematuros de menor edad gestacional (EG). Parte de ellos nacen con una restricción de crecimiento intrauterino $(\mathrm{RCIU})^{2}$. La posibilidad de recuperar o mantener un crecimiento restringido depende de las causas y magnitud de la RCIU, la morbilidad y manejo nutricional posterior ${ }^{2}$. A pesar de los avances en nutrición y la mejoría en los aportes en macro y micronutrientes, es frecuente que durante la hospitalización se agregue un crecimiento postnatal insuficien$\mathrm{te}^{3,4}$. Un peso menor del percentil 10 al alta se ha denominado restricción del crecimiento postnatal (RCPN), y se puede observar en niños con buen crecimiento prenatal o con RCIU ${ }^{3}$.

Numerosos estudios muestran la importancia de una buena evolución nutricional en el neurodesarrollo desde las primeras semanas de vida ${ }^{5-9}$. Lograr un crecimiento cercano al crecimiento fetal es una de las tareas importantes en Neonatología.

La recuperación de peso hasta los 3 a 4 meses de edad corregida (EC), se asocia con efectos significati- 
vos en el neurodesarrollo, pero posterior a esta edad aumenta riesgo de obesidad ${ }^{10}$. El crecimiento craneano hasta los 8 meses se ha asociado claramente a mejor desarrollo ${ }^{11}$.

La RCIU es un proceso prenatal, de seguimiento obstétrico, que puede manejarse con interrupción del embarazo determinando prematurez. Con las curvas de crecimiento intrauterino se clasifica según el peso y la edad gestacional al nacer al recién nacido bajo percentil 10 como Pequeño para la Edad Gestacional (PEG) y sobre el percentil 10 y bajo el 90 como Adecuados para la Edad Gestacional $(\mathrm{AEG})^{2}$. El seguimiento nutricional con puntaje $\mathrm{Z}$, corresponde a la diferencia en desviación estándar de la media para edad y sexo. Si la curva de distribución de la población es normal, el percentil 10 de una curva corresponde a $-1,3$ desviaciones o un puntaje $\mathrm{Z}$ de $-1,3^{12}$.

El Programa de Alimentación Complementaria del Prematuro (PNACP) de Chile, entrega formulas especiales durante el primer año de vida a todos los prematuros de menos de $1500 \mathrm{~g}$ o de menos de 32 semanas al nacer hasta los 12 meses de edad corregida ${ }^{13}$. Evaluar e intervenir el crecimiento postalta es una de las actividades de los Programas de Seguimiento de los nacidos prematuros o de muy bajo peso ${ }^{14}$.

El objetivo de este estudio es describir la evolución antropométrica de recién nacidos prematuros menores de 32 semanas, desde el nacimiento hasta los 24 meses de EC.

\section{Pacientes y Método}

Estudio observacional, multicéntrico, descriptivo y retrospectivo sobre el crecimiento de recién nacidos prematuros controlados en 5 Policlínicos de Seguimiento de Prematuros del Sistema Público de Salud en la región central de Chile, egresados de su hospitalización neonatal durante los años 2011 y 2012.

En este estudio se incluyeron 721 lactantes nacidos con menos de 32 semanas de edad gestacional, que tuvieron información completa al nacer, alta, 40 semanas y entre 18 y 24 meses de edad corregida. Se excluyeron pacientes con genopatías o con diagnósticos congénitos que comprometen el crecimiento. Los pacientes fueron controlados en los Policlínico de cada centro. El Programa de Seguimiento tenía un protocolo y criterio común de evaluación en cada control. Se clasificó a cada lactante según estado nutricional al nacer, al alta y a las 40 semanas con curvas de Fenton que distingue por sexo y edad gestacional exacta (semanas y días) y posteriormente según curvas de crecimiento OMS con programa Anthro ${ }^{15,16}$.

Los lactantes prematuros se clasificaron en 4 grupos:
1. Grupo AAA: lactantes nacidos AEG, sin restricción postnatal (al nacer, al alta y 40 semanas con percentil de peso mayor de 10).

2. Grupo APA: lactantes nacidos AEG con restricción postnatal, con peso al alta menor del $10 \mathrm{y}$ a las 40 semanas con peso mayor de percentil de 10 .

3. Grupo APP: lactantes nacidos AEG, con restricción postnatal, con peso al alta en percentil menor de 10 , que se mantiene bajo percentil 10 a las 40 semanas).

4. Grupo PPP: lactantes nacidos con restricción intrauterina y que mantuvieron restricción postnatal (al nacer, al alta y 40 semanas con peso bajo el percentil 10).

Se obtuvieron los datos de peso, longitud y perímetro cefálico al nacer, al alta, a las 40 semanas, y al mes 1 , $3,6,9,12,18$ y 24 meses de EC, sexo, edad gestacional al nacer y duración de hospitalización de las fichas de cada centro de seguimiento. Las mediciones antropométricas post alta fueron realizadas en cada policlínico de seguimiento en su control clínico, peso con balanza calibrada, longitud en decúbito supino entre 2 personas, con podómetro y el perímetro de cráneo con el mayor diámetro occipitofrontal con cinta métrica no extensible.

Los datos al nacer y al alta fueron evaluados con edad exacta. El control de 1 mes se considera con una variación de más o menos 15 días y en los controles siguientes se consideraron variaciones de la fecha del control $\pm 1,5$ meses, ya que la mayoría de los controles no fueron realizados en la fecha exacta que les correspondía. El cálculo de puntaje $Z$ se realizó con la edad exacta al día del control.

Se describió evolución de peso, longitud y perímetro craneano para cada edad. Se comparó evolución de los grupos: AAA, APA, APP, PPP con puntaje Z considerando bajo peso un $\mathrm{Z}$ menor de -1,3. El puntaje $\mathrm{Z}$ de la relación peso/talla se describe desde las 40 semanas.

\section{Análisis estadístico}

Se calcularon los puntajes Z para los distintos índices antropométricos con el programa Fenton hasta las 40 semanas y Anthro para los controles posteriores ${ }^{13,15}$. Las tendencias de crecimiento se evaluaron utilizando valores de puntuación $\mathrm{Z}$ para el peso, la longitud, perímetro craneano y peso para la talla desde el nacimiento hasta los 2 años de edad corregida. Los análisis se realizaron entre los grupos según patrón de crecimiento (AAA, APA, APP y PPP).

Para la descripción de las variables continuas se utilizó, según su distribución, medidas de tendencia central (promedio y mediana) y medidas de dispersión absoluta (desviación estándar y rango intercuartílico). 
En el caso de las variables categóricas se describieron mediante frecuencias absolutas y relativas. Las diferencias entre los grupos se analizaron mediante ANOVA para muestras independientes y por Kruskal Wallis. Para las variables categóricas se utilizaron pruebas de Chi-cuadrado. Para el análisis de medidas repetidas se utilizaron Modelos Lineales Mixtos, donde se usó como variable dependiente a cada índice antropométrico (puntaje Z) y como variable explicativa el grupo según patrón de crecimiento. Además, se realizaron subanálisis por intervalo de tiempo para ver las diferencias entre los grupos en los primeros seis meses de edad corregida y luego desde los seis hasta los dos años de edad corregida. La significancia se fijó en 0.05. Para los análisis se utilizaron EpiInfo, Excel, STATA 13.0 SE para MAC (Lakeway Drive College Station, TX, EE. UU.)

El estudio fue aprobado por el Comité de Ética de Investigación del Servicio de Salud Metropolitano SurOriente, en Santiago, el 9 de marzo de 2021. Los datos fueron tratados anónimamente.

\section{Resultados}

Se incluyeron 721 prematuros que tuvieron control desde las 40 semanas de EC. 321 (44,5\%) prematuros fueron de sexo femenino. Destaca que 213 (29,5\%) egresaron de la hospitalización neonatal con peso menor a $Z-1,3$ y $508(70,5 \%)$ con peso mayor o igual a Z-1,3. La mayoría (537) tuvo control a los 24 meses En la figura 1 se detalló la distribución de grupo según estado nutricional al nacer, al alta y a las 40 semanas.

La tabla 1 muestra la antropometría al nacer, al alta, a las 40 semanas y a los 24 meses de los grupos estudiados. Hubo diferencias significativas en todas las variables, excepto en sexo. Entre los dos grupos de peso sobre $Z-1,3$ a las 40 semanas (AAA y APA), las diferencias se observaron en antropometría al alta y edad gestacional al egreso hospitalario. Los dos grupos con peso bajo Z -1,3 (PPP y APP) fueron significativamente diferentes en la antropometría al nacer, peso y longitud a las 40 semanas, con similar edad gestacional al nacer y al egreso de hospitalización, como se observa en la tabla 2.

En la figura 2 se observa la evolución del promedio del puntaje $\mathrm{Z}$ de peso ( $\mathrm{ZP}$ ) desde el nacimiento hasta los 2 años de EC. El grupo AAA, sin restricción de crecimiento postnatal, tuvo una evolución de $\mathrm{ZP}$ a las 40 semanas de edad gestacional cercana a $Z+0,5$, desde los 3 meses se ubicó en la mediana y se mantuvo así hasta los 24 meses de EC. El grupo APA presentó una marcada RCPN, pero se recupera aceleradamente y sigue un trayecto similar al grupo AAA. Las diferencias entre estos dos grupos en puntaje $Z$ de peso no fueron significativas desde los 6 meses. Se examinó la evolu-
Figura 1. Distribución de la muestra analizada. AAA: peso al nacer, al alta y 40s sobre el percentil 10. APA: peso al nacer y a las 40 semanas sobre el p10 y al alta bajo el percentil 10. APP: peso al nacer sobre el p10 y al alta y 40 semanas bajo el p10. PPP: peso al nacer, al alta y 40 s bajo el percentil 10 .

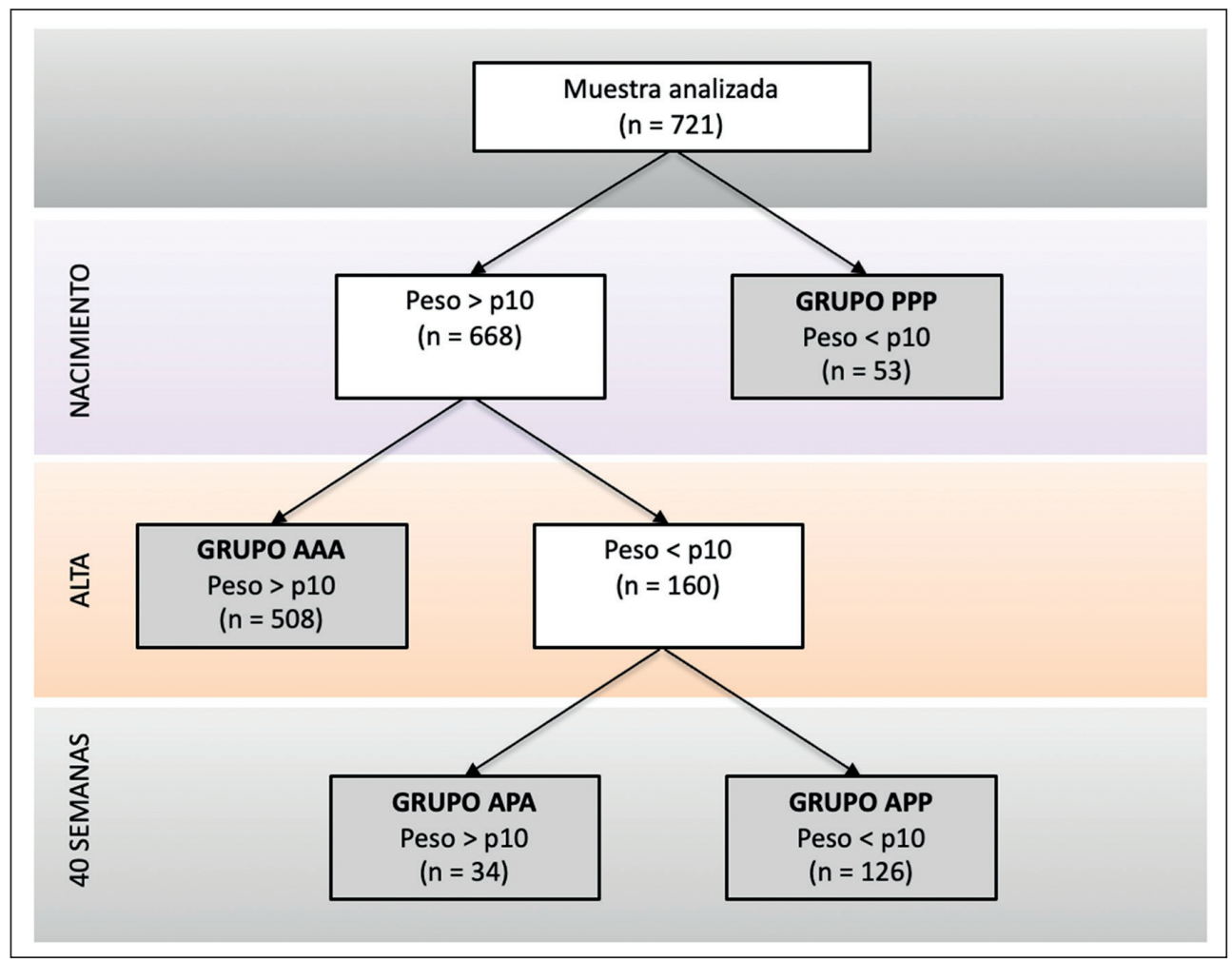


Tabla 1. Características antropométricas al nacer, al alta, a las 40 semanas postnatal y dos años de edad corregida de los cuatro grupos estudiados

\begin{tabular}{|c|c|c|c|c|c|}
\hline & AAA(a) & $\mathrm{APA}(\mathrm{b})$ & $\operatorname{APP}(c)$ & $\operatorname{PPP}(d)$ & $p$ \\
\hline $\mathrm{n} / \%$ & $508 / 70,5 \%$ & $34 / 5,4 \%$ & $126 / 17,5 \%$ & $53 / 7,4 \%$ & Diferencias $p<0,05$ \\
\hline Peso nacer g & $1376(332)$ & 1317 (305) & $1030(260)$ & $817(188)$ & $\begin{aligned} a, b & >c, d \\
c & >d\end{aligned}$ \\
\hline Longitud nacer cm & $38,7(3,1)$ & $37,9(3,4)$ & $35,4(3,3)$ & $33,3(3)$ & $\begin{array}{c}a, b>c, d \\
c>d\end{array}$ \\
\hline Perímetro cráneo cm & $27,7(2,2)$ & $27,5(1,8)$ & $24,4(2,3)$ & $24,8(1,9)$ & $a, b>c, d$ \\
\hline Edad gestacional, sem & $29,1(1,8)$ & $29,6(1,4)$ & $28,2(2,1)$ & $28,8(1,9)$ & $a, b>c, d$ \\
\hline$\%$ Femenino & 44,7 & 32,1 & 50 & 38,2 & Ns \\
\hline \multicolumn{6}{|l|}{ Alta } \\
\hline Semanas hospitalizado* & $7,3(5,4-10,9)$ & $8,3(5,9-10,7)$ & $11(8,1-15,4)$ & $11,6(8,4-14,3)$ & $a, b<c, d$ \\
\hline Peso g & $2855(726)$ & 2447 (488) & $2640(529)$ & $2574(572)$ & $a,>b, c, d$ \\
\hline Longitud cm & $47,2(3,1)$ & $45,5(2,7)$ & $46,5(2,8)$ & $45,5(3,1)$ & $a>, b, c, d$ \\
\hline Perímetro cráneo cm & $33,8(2,2)$ & $32,5(2,2)$ & $33,5(1,8)$ & $33,4(1,8)$ & $b<$ a.c.d \\
\hline Edad gestacional & $37,6(3)$ & $38,7(3,1)$ & $40(3,1)$ & $40,5(2,9)$ & $a, b<c, d$ \\
\hline \multicolumn{6}{|l|}{40 semanas } \\
\hline Peso g & 3521 (474) & 3475 (397) & $2771(237)$ & $2601(363)$ & $a, b>c, d$ \\
\hline Longitud cm & $49,7(4,3)$ & $49,6(1,9)$ & $46,6(1,6)$ & $45,3(2,3)$ & $a, b>c, d$ \\
\hline Perímetro craneano cm & $35,9(1,4)$ & $35,9(1,1)$ & $34,4(1,8)$ & $34(1,3)$ & $a, b>c, d$ \\
\hline Peso /Talla & $0,70(1,03)$ & $0,63(0,89)$ & $0,02(0,96)$ & $0,09(0,73)$ & $a, b>c, d$ \\
\hline \multicolumn{6}{|l|}{24 meses } \\
\hline Peso K & $12,22(1,59)$ & $12,50(2,24$ & $10,84(1,32)$ & $10,53(1,27)$ & $a, b>c, d$ \\
\hline Longitud cm & $86,6(3,4)$ & $87,8(3,7)$ & $83,9(3,4)$ & $83,4(3,6)$ & $a, b>c, d$ \\
\hline Perímetro craneano cm & $48,7(1,9)$ & $48,8(1,9)$ & $47,2(1,9)$ & $47,1(1,8)$ & $a, b>c, d$ \\
\hline Peso/Talla & $0,24(1,04)$ & $0,13(1,33)$ & $-0,49(1,04)$ & $-0,81(0,96)$ & $a, b>c, d$ \\
\hline
\end{tabular}

AAA: peso al nacer, al alta y 40s sobre el percentil 10 (a); APA: peso al nacer y a las 40 semanas sobre el p10, al alta bajo el percentil 10 (b); APP: peso al nacer sobre el p10, al alta y 40 semanas bajo el p10 (c); PPP: peso al nacer, al alta y 40s bajo el percentil 10 (d) Datos en promedio y ds. *Mediana y rango intercuartílico.

ción entre el alta y las 40 semanas en el grupo APA buscando interpretar estos datos: 4 casos de este grupo tuvieron alta después de las 40 semanas, una parte de los casos tuvo alta precoz a un segundo centro donde tuvieron crecimiento de recuperación. El incremento promedio entre el alta y las 40 semanas en el grupo fue de $39 \mathrm{~g}$ diarios.

Los otros dos grupos con RCPN tuvieron una evolución similar, pero el grupo APP en un nivel mayor entre 0,5 y 0,8 desviaciones estándar. A las 40 semanas el grupo PPP tuvo un promedio de ZP de -2,3 y el grupo APP de $-1,8$, diferencia estadísticamente significativa.

En la figura 3 se observa un puntaje $Z$ de longitud (ZL) con una curva de evolución similar al peso, pero más alejada de la mediana, mostrando el mayor compromiso de la longitud durante la evolución. Los dos grupos adecuados al nacer y 40 semanas (AAA y APA) tuvieron una evolución diferente desde el nacimiento hasta los 3 meses de EC, sin diferencias significativas

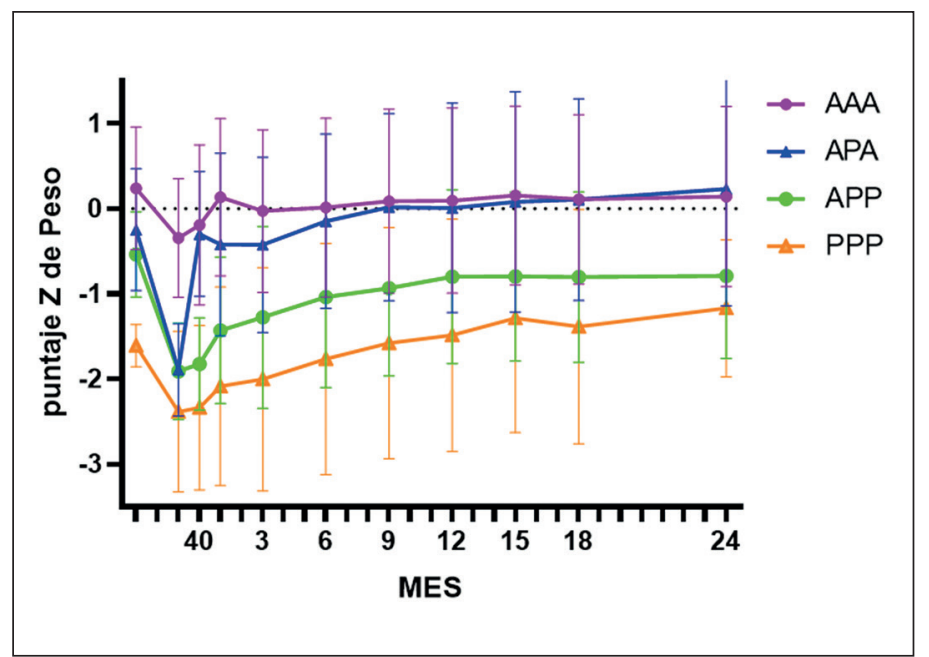

Figura 2. Puntaje $Z$ de peso desde nacimiento a 24 meses de edad corregida por grupo, promedio \pm ds. AAA: peso al nacer, al alta y 40 s sobre el percentil 10 (a); APA: peso al nacer y a las 40 semanas sobre el p10, al alta bajo el percentil 10 (b); APP: peso al nacer sobre el p10, al alta y 40 semanas bajo el p10 (c); PPP: peso al nacer, al alta y 40s bajo el percentil 10. 
posteriormente. El grupo con RCIU y postnatal (PPP) presentó promedio más bajo de ZL de -2,8, al mes de EC, posteriormente presentó crecimiento recuperacional en longitud y a los 24 meses tuvo un ZL de -1,16. Por otro lado, el grupo APP recuperó estatura a ese nivel a los 9 meses de EC, y a los 24 meses tuvo ZL sobre -1 .

La figura 4 de puntaje $\mathrm{Z}$ de perímetro craneano (ZPC) presentó una curva similar a la del peso, pero mucho más estrecha. Sólo hay diferencias entre el nacimiento y las 40 semanas de EC en los grupos con buen

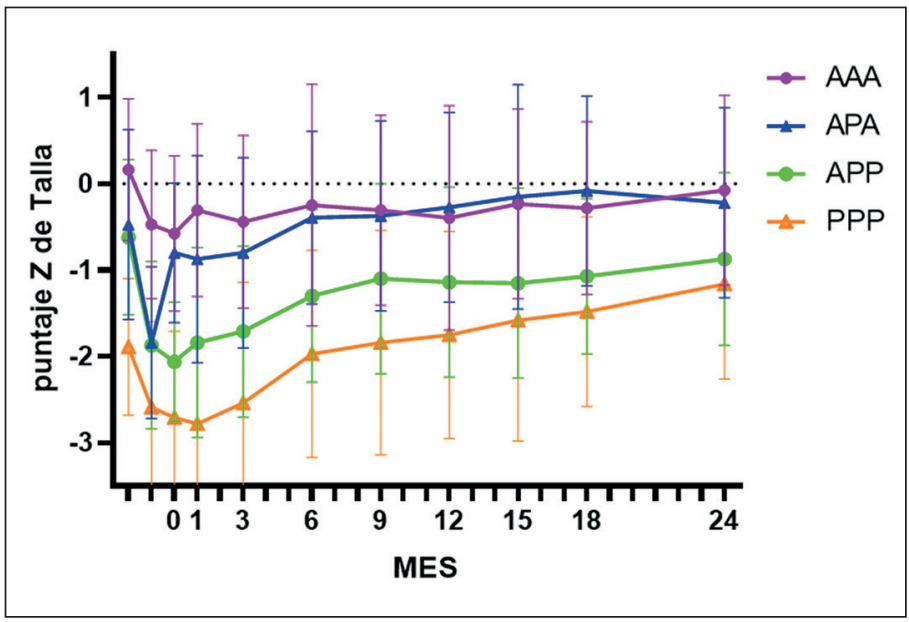

Figura 3. Puntaje $Z$ de talla desde nacimiento a 24 meses de edad corregida por grupo, promedio \pm ds. AAA: peso al nacer, al alta y 40 s sobre el percentil 10 (a); APA: peso al nacer y a las 40 semanas sobre el p10, al alta bajo el percentil 10 (b); APP: peso al nacer sobre el p10, al alta y 40 semanas bajo el p10 (c); PPP: peso al nacer, al alta y 40s bajo el percentil 10.

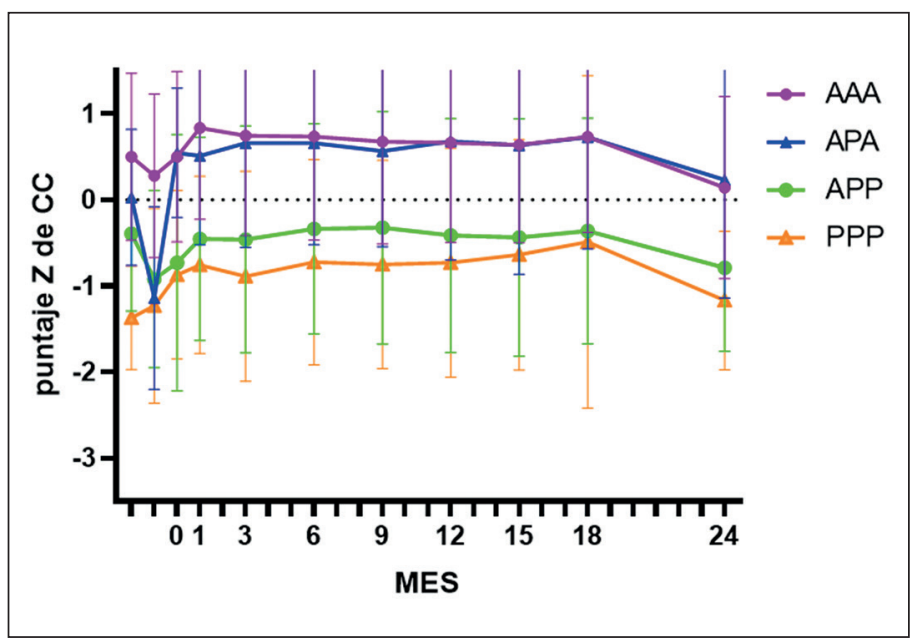

Figura 4. Puntaje $Z$ de Circunferencia craneana desde el nacimiento a los 24 meses de edad corregida por grupo, promedio $\pm d s$. AAA: peso al nacer, al alta y 40 s sobre el percentil 10 (a); APA: peso al nacer y a las 40 semanas sobre el p10, al alta bajo el percentil 10 (b); APP: peso al nacer sobre el p10, al alta y 40 semanas bajo el p10 (c); PPP: peso al nacer, al alta y 40 s bajo el percentil 10. estado nutricional a las 40 semanas. El grupo AAA al alta y a las 40 semanas se ubica en el canal que mantiene los dos primeros años. El grupo APA se ubica entre 40 semanas y un mes en la curva que continúa hasta los 24 meses de EC. El menor nivel lo presentó el grupo PPP al nacer con un valor de -1,37 y recuperó a los 18 meses a ZPC -0,5. El grupo APP estabilizó su ZPC a los 6 meses de EC. Los tres gráficos tienen las mismas unidades en el eje $\mathrm{Y}$, para observar más claramente las diferencias de evolución de los tres parámetros antropométricos.

La figura 5 muestra la evolución del puntaje $\mathrm{Z}$ de $\mathrm{P} / \mathrm{T}$, con un patrón común en los primeros 3 meses para los 4 grupos y posteriormente una marcada caída para el grupo PPP que pese a recuperar gradualmente el peso y la talla se mantiene con un $\mathrm{P} / \mathrm{T}$ muy bajo.

En las tablas 2 y 3 se muestra el análisis de cada medida antropométrica por modelos lineales mixtos en dos periodos: desde 0 a 6 meses y de 6 a 24 meses. En el modelo de 0 a 6 meses todos los grupos son diferentes de AAA, salvo en peso/talla que APA no es diferente de AAA. En el modelo de 6 a 24 meses no hay diferencia entre AAA y APA, pero ambos son diferentes de APP y PPP.

\section{Discusión}

En este estudio analizamos la evolución antropométrica desde el nacimiento hasta los 2 años de edad corregida de 4 grupos de prematuros de menos de 32 semanas al nacer, observando que el grupo con buen crecimiento prenatal e intrahospitalario mantiene evolución similar a la mediana de referencia OMS posterior a las 40 semanas, con ZP calculado por Anthro, con EC hasta los 24 meses.

Los lactantes que presentaron RCPN se distribuyen en 3 grupos: 2 grupos nacen con peso sobre el percentil 10 de la curva de Fenton, uno de los cuales presenta crecimiento recuperacional y presenta un $\mathrm{ZP}$ mayor de $(-1,3)$ a las 40 semanas (grupo APA), y otro que mantiene un crecimiento insuficiente (grupo APP), pero inicia desde el alta una recuperación gradual de peso, alcanzando ZP (-1) a los 6 meses de EC y ZP $(-0,8)$ a los 12 meses que se mantiene por el segundo año.

La mediana del perímetro craneano no resulta comprometida en ninguno de los grupos, siendo mayor de Z -1,3 (equivalente al percentil 10) a las 40 semanas postconcepcional, ilustrando la prioridad del crecimiento cerebral.

Estudios previos han considerado al prematuro con RCIU como el grupo con mayor riesgo de déficit de crecimiento $^{19}$. Este estudio agrega que también los prematuros con buen crecimiento prenatal pero con restricción de crecimiento post natal, pueden tener un 
Tabla 2. Diferencias en puntaje $Z$ en peso, talla y CC para edad, y peso para talla de 0 a 6 meses de edad

\begin{tabular}{|c|c|c|c|c|}
\hline & Grupo & Coeficiente & Intervalo Confianza 95\% & $\mathrm{p}$ \\
\hline \multirow[t]{5}{*}{$Z$ peso /edad } & AAA ref & & & \\
\hline & APA & $-0,53$ & $(-0,77--0,30)$ & 0,000 \\
\hline & APP & $-1,29$ & $(-1,42--1,15)$ & 0,000 \\
\hline & PPP & $-2,00$ & $(-2,19--1,80)$ & 0,000 \\
\hline & cons & $-0,032$ & $(-0,09-0,03)$ & 0,292 \\
\hline \multirow[t]{5}{*}{ Z talla / edad } & AAA ref & & & \\
\hline & APA & $-0,48$ & $(-0,74--0,23)$ & 0,000 \\
\hline & APP & $-1,16$ & $(-1,30--1,01)$ & 0,000 \\
\hline & PPP & $-2,03$ & $(-2,24--1,82)$ & 0,000 \\
\hline & cons & $-0,38$ & $(-0,45--0,32)$ & 0,000 \\
\hline \multirow[t]{5}{*}{ Z circ/ edad } & AAA ref & & & \\
\hline & APA & $-0,45$ & $(-0,71--0,18)$ & 0,000 \\
\hline & APP & $-1,13$ & $(-1,28--0,98)$ & 0,000 \\
\hline & PPP & $-1,59$ & $(-1,81--1,37)$ & 0,001 \\
\hline & cons & 0,57 & $(0,498-0,633)$ & 0,000 \\
\hline \multirow[t]{5}{*}{ Z peso/ talla } & AAA ref & & & \\
\hline & APA & $-0,48$ & $(-0,74--0,23)$ & 0,000 \\
\hline & APP & $-1,16$ & $(-1,30--1,01)$ & 0,000 \\
\hline & PPP & $-2,03$ & $(-2,25--1,82)$ & 0,000 \\
\hline & cons & $-0,38$ & $(-0,45--0,32)$ & 0,000 \\
\hline
\end{tabular}

ref: referencia cons: constante. AAA: peso al nacer, al alta y 40s sobre el percentil 10 (a); APA: peso al nacer y a las 40 semanas sobre el p10, al alta bajo el percentil 10 (b); APP: peso al nacer sobre el p10, al alta y 40 semanas bajo el p10 (c); PPP: peso al nacer, al alta y 40 s bajo el percentil 10 .

Tabla 3. Diferencias en puntaje $Z$ en peso, talla y CC para edad, y peso para talla de 6 a 24 meses de edad

\begin{tabular}{|c|c|c|c|c|}
\hline & Grupo & Coeficiente & Intervalo Confianza 95\% & $p$ \\
\hline \multirow[t]{5}{*}{ Z peso /edad } & AAA ref & & & \\
\hline & APA & $-0,10$ & $(-0,46-0,26)$ & 0,585 \\
\hline & APP & $-0,93$ & $(-1,13--0,72)$ & 0,000 \\
\hline & PPP & $-1,58$ & $(-1,88--1,28)$ & 0,000 \\
\hline & cons & 0,06 & $(-0,03-0,15)$ & 0,188 \\
\hline \multirow[t]{5}{*}{ Z talla / edad } & AAA ref & & & \\
\hline & APA & $-0,25$ & $(-0,53-0,02)$ & 0,070 \\
\hline & APP & $-1,0$ & $(-1,15--0,84)$ & 0,000 \\
\hline & PPP & $-1,74$ & $(-1,97--1,52)$ & 0,000 \\
\hline & cons & $-0,37$ & $(-0,44--0,30)$ & 0,000 \\
\hline \multirow[t]{5}{*}{ Z circ/ edad } & AAA ref & & & \\
\hline & APA & 0,0004 & $(-0,39-0,40)$ & 0,998 \\
\hline & APP & $-1,04$ & $(-1,27--0,82)$ & 0,000 \\
\hline & PPP & $-1,35$ & $(1,68--1,03)$ & 0,000 \\
\hline & cons & 0,67 & $(0,57-0,77)$ & 0,000 \\
\hline \multirow[t]{5}{*}{ Z peso/ talla } & AAA ref & & & \\
\hline & APA & $-0,16$ & $(-0,51-0,18)$ & 0,355 \\
\hline & APP & $-0,69$ & $(-0,88--0,49)$ & 0,000 \\
\hline & PPP & $-1,08$ & $(-1,37--0,80)$ & 0,000 \\
\hline & cons & 0,34 & $(0,26-0,43)$ & 0,000 \\
\hline
\end{tabular}

ref: referencia cons:constante. AAA: peso al nacer, al alta y 40 s sobre el percentil 10 (a); APA: peso al nacer y a las 40 semanas sobre el p10, al alta bajo el percentil 10 (b); APP peso al nacer sobre el p10, al alta y 40 semanas bajo el p10 (c); PPP: peso al nacer, al alta y 40 s bajo el percentil 10. 


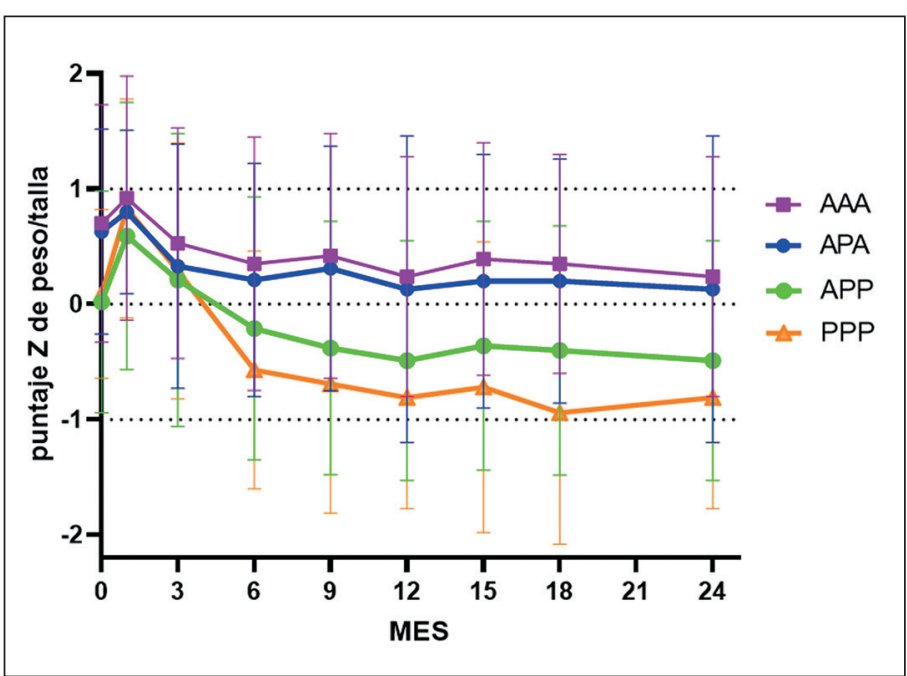

Figura 5. Puntaje $Z$ de Peso para talla desde las 40 semanas a los 24 meses de edad corregida por grupo, promedio \pm ds. AAA: peso al nacer, al alta y $40 \mathrm{~s}$ sobre el percentil 10 (a); APA: peso al nacer y a las 40 semanas sobre el p10, al alta bajo el percentil 10 (b); APP: peso al nacer sobre el p10, al alta y 40 semanas bajo el p10 (c); PPP: peso al nacer, al alta y 40s bajo el percentil 10

crecimiento recuperacional a las 40 semanas de EC, o pueden mantener una lenta recuperación con curva similar al prematuro con RCIU, pero menos comprometida.

La incidencia de RCPN en esta población fue de $19,3 \%$ con un $7,4 \%$ que ya tenía RCIU (tabla 1 ). Revisando datos de la literatura estas cifras son comparables $^{20}$. Las definiciones de lo que se considera RCPN son variadas, inicialmente fueron menor del p10 pero con el uso de puntaje $Z$ se ha propuesto cortes en -1, $-1,5$ y - $2^{21-24}$ También se ha utilizado la diferencia entre el puntaje $\mathrm{Z}$ al nacer $\mathrm{y}$ al alta, se han propuesto cifras de disminución del puntaje de 0,8 o de $1^{21,22}$. Falta consensuar una definición con base fisiopatológica ${ }^{21}$. La otra variable que influye es la curva de referencia utilizada. Nosotros utilizamos Fenton y Anthro pragmáticamente porque existían los software adecuados, pero si utilizáramos las actuales curvas en uso en Chile el porcentaje de RCIU y de RCPN sería mayor ${ }^{25}$. Se citan cifras de RCPN entre 26 y $59 \%$ con diferentes curvas $^{26}$. Las curvas Intergrowth podrían en el futuro solucionar la heterogeneidad de curvas y permitir comparar distintas poblaciones más adecuadamen$\mathrm{te}^{26}$.

También se ha distinguido el real RCPN como aquel que no tiene RCIU y que el deterioro nutricional se produce durante la hospitalización ${ }^{27}$. Este grupo constituye el $22,9 \%$ en nuestros datos (tabla 1). Además del crecimiento prenatal influyen durante la hospitalización la precocidad del aporte nutricional, especialmente un buen aporte de aminoácidos desde el primer día ${ }^{5-7}$. Entre la morbilidad que afecta el cre- cimiento se nombran la displasia broncopulmonar, sepsis tardía y enterocolitis necrotizante especialmente quirúrgica ${ }^{28,29}$.

Respecto a la evolución de longitud, la caída del puntaje $\mathrm{Z}$ continúa post alta y en el grupo PPP se observa un promedio de $-2,75$ pasado el mes de edad corregida, después hay un lento crecimiento de longitud, hasta los 24 meses.

La circunferencia craneana se recupera rápidamente en todos los casos. Un crecimiento adecuado desde el periodo intrauterino y postnatal en el seguimiento es una base de sustentación para posibilitar un desarrollo cerebral cercano a lo normal, y una evolución neurocognitiva favorable $e^{30}$.

La evolución del puntaje $\mathrm{Z}$ de relación peso/talla presenta una curva muy interesante: al mes de edad corregida la diferencia entre los 4 grupos no es significativa y la mayor diferencia es de 0,24 unidades $\mathrm{Z}$ (desviación estándar). A los 3 meses hay una diferencia significativa entre el grupo AAA y el PPP, pero de 0,32 unidades $\mathrm{Z}$. A los 6 meses el puntaje $\mathrm{Z} \mathrm{P} / \mathrm{T}$ cae bajo la mediana en los grupos APP, PPP, mientras que los otros dos grupos AAA y APA se mantiene sobre la mediana. El grupo PPP cae a los 6 meses a $-0,57$ y sigue cayendo levemente hasta los 18 meses a $-0,94$. Clínicamente estas cifras se manifiestan en el aspecto de sobrepeso que estos prematuros tienen entre 1 y 3 meses, con relaciones $\mathrm{P} / \mathrm{T}$ sobre la mediana, pero posteriormente no suben igual de peso, y la relación $\mathrm{P} / \mathrm{T}$ en AAA y APA se mantiene normal, pero los lactantes con antecedentes de RCIU o RCPN sin rápida recuperación tienen un menor incremento de peso, mientras la longitud sube lentamente, y especialmente los con RCIU tienden a observarse adelgazados. En estudios de composición corporal estos niños mantienen tanto masa grasa, como libre de grasa mucho menor que controles de peso y edad de nacimiento normal ${ }^{31}$.

La evolución de peso posterior al alta ha perdido valor predictor, cuando se controla por longitud y $\mathrm{PC}^{5}$. Longitud y PC son más sensibles y específicos asociados a mejor desarrollo neurocognitivo, aunque también es afectado por la morbilidad y el manejo nutricional $^{5,27-29}$. Sin embargo, el peso sigue siendo una referencia clínica ya que la evolución antropométrica tiene interdependencia.

En nuestros datos no encontramos diferencia en evolución antropométrica por puntaje $\mathrm{Z}$ entre niños y niñas (datos no se muestran). Habitualmente las niñas frente a suplementos nutricionales, responden menos en antropometría y desarrollo cognitivo, pero basalmente están mejor que los niños ${ }^{32,33}$.

$\mathrm{Al}$ alta estos lactantes reciben fórmula láctea para prematuros como complemento a la lactancia materna, hasta los 6 meses de edad corregida, y una fórmula láctea infantil para el segundo semestre según 
el PNACP ${ }^{13}$. La disponibilidad de alimentación láctea permite asumir que no hay un factor primario, importante en la evolución. La alimentación post alta de prematuros no ha originado consenso. En la literatura se ha probado una fórmula de transición para después del alta, que tiene una composición intermedia entre una fórmula para prematuro y una fórmula de inicio. Algunos estudios mantienen fórmula para prematuros hasta las 40 semanas de EC y posteriormente continúan con una formula láctea de inicio ${ }^{34}$. Se han propuesto fórmulas especiales con mayor aporte de proteínas y micronutrientes considerando que el lactante regula ingesta oral por concentración de energía y que la recuperación de masa magra debe ser mayor que la grasa $^{35,36}$.

El termino de desnutrición postnatal o restricción de crecimiento postnatal permite comparar evolución de diferentes centros, aunque ha sido cuestionado recientemente por la baja especificidad de la evolución del peso solamente en el desarrollo cognitivo al usarlo como predictor de un individuo ${ }^{19}$. La longitud y especialmente el perímetro craneano tienen mejor sensibilidad y especificidad al respecto. La longitud es más difícil de medir apropiadamente, de manera que un mayor PC con una neuroimagen normal es más útil y tiene mejor pronóstico ${ }^{37}$.

Las comparaciones entre prematuros con RCIU y sin RCIU dependen de si se hacen por peso, por ejemplo menores de $1.500 \mathrm{~g}$ al nacer o por edad gestacional $^{38}$. En este caso hemos agregado a los casos con RCPN, la comparación de recuperación precoz o no, y se destaca la diferencia importante entre los con RCPN que se recuperan y no; y lo parecido que evolucionan los lactantes con antecedentes RCIU y RCPN (grupo $\mathrm{PPP})$, con los que sólo han tenido restricción postnatal.

La principal fortaleza de este estudio es el número de pacientes estudiados, provenientes de varios centros de seguimiento, con un plan de atención común, protocolizado y un programa de alimentación complementaria durante el primer año ${ }^{14}$.

Habría sido muy interesante contar con la evolución de crecimiento y el aporte nutricional durante la hospitalización y las principales morbilidades presentadas para incluirlas en estudio de factores asociados a la evolución posterior. Por otra parte, haber contado con una estimación de desarrollo neurocognitivo a los dos años de estos 4 grupos permitiría ver cuán asociado están estos patrones de crecimiento al desarrollo posterior. Otra limitante es que la asistencia no es en la fecha exacta, pero el puntaje $\mathrm{Z}$ se asigna por edad exacta, con lo que compensa esta situación.

La nutrición óptima, intensiva y oportuna, es un factor crítico y debe ser un objetivo importante en el período intrahospitalario ya que es determinante en el crecimiento posterior $^{5-9}$. En nuestro estudio se destaca que el crecimiento precoz está asociado a la evolución antropométrica posterior.

\section{Conclusiones}

La evolución de crecimiento de prematuros nacidos con menos de 32 semanas de edad gestacional es variada durante la hospitalización y después del alta. Un peso mayor del percentil 10 a las 40 semanas se asocia a un buen crecimiento en los 2 primeros años de vida, por lo que éste constituye un objetivo importante de la nutrición neonatal cuando se ha presentado previamente una evolución nutricional desfavorable. Analizar la información de la morbilidad y manejo durante la hospitalización permitiría identificar factores clínicos asociados a un buen peso a las 40 semanas y programar intervenciones clínicas durante la hospitalización para minimizar el riesgo de desnutrición postnatal en prematuros menores de 32 semanas.

\section{Responsabilidades Éticas}

Protección de personas y animales: Los autores declaran que los procedimientos seguidos se conformaron a las normas éticas del comité de experimentación humana responsable y de acuerdo con la Asociación Médica Mundial y la Declaración de Helsinki.

Confidencialidad de los datos: Los autores declaran que han seguido los protocolos de su centro de trabajo sobre la publicación de datos de pacientes.

Derecho a la privacidad y consentimiento informado: Los autores han obtenido el consentimiento informado de los pacientes y/o sujetos referidos en el artículo. Este documento obra en poder del autor de correspondencia.

\section{Conflicto de intereses}

Los autores declaran no tener conflicto de intereses. 


\section{Referencias}

1. Helenius K, Sjors G, Shah PS, et al. Survival in Very Preterm Infants: An International Comparison of 10 National Neonatal Networks. Pediatrics 2017;140:e20171264.

2. Carducci B, Bhutta Z, Care of the Growth Restricted Newborn. Best Pract Res Clin Obstet Gynaecol. 2018;49:103-16.

3. Clark RH, Thomas P, Peabody J: Extrauterine growth restriction remains a serious problem in prematurely born neonates. Pediatrics 2003;111:986-90.

4. Wit JM, Finken M, Rijken M, et al. Preterm Growth Restraint: A Paradigm that Unifies Intrauterine Growth Retardation and Preterm Extrauterine Growth retardation and has implications for the Small for Gestational Age Indication in Growth Hormone Therapy. Pediatrics 2006;117:793-5.

5. Ramel S, Gray H, Christiansen E, et al. Greater early gains in fat-free mass, but not fat mass, are associated with improved neurodevelopmental lyear corrected age for prematurity in very low birth weight preterm infants. J Pediatr. 2016;173:108-15.

6. Ruys CA, Hollanders JJ, Bröring T, et al. Early-life growth of preterm infants and its impact on neurodevelopment. Pediatr Res. 2019;85:283-92.

7. Franz AR, Pohlandt F, Bode $\mathrm{H}$, et al. Intrauterine, early neonatal, and postdischarge growth and neurodevelopmental outcome at 5.4 years in extremely preterm infants after intensive neonatal nutritional support. Pediatrics 2009;123:e101-e109.

8. Sammallahti S, Kajantie E, Matinolli HM, et al. Nutrition after preterm birth and adult neurocognitive outcomes. PLoS One. 2017;12:e185632.

9. Egashira T, Hashimoto M, Shiraishi TA, et al. A longer body length and larger head circumference at term significantly influences a better subsequent psychomotor development in verylow-birth-weight infants. Brain Dev. 2019;41(4):313-9.

10. Belfort MB, Rifas-Shiman SL, Sullivan T, et al. Infant growth before and after term: efects on neurodevelopment in preterm infants. Pediatrics 2011;128:e899-906.

11. Hack M, Breslau N, Weissman B, et al. Effect of very low birth weight and subnormal head size on cognitive abilities at school age. N Engl J Med. 1991;325:231-7.

12. Fenton TR, Sauve RS. Using the LMS method to calculate $\mathrm{z}$-scores for the Fenton preterm infant growth chart. Eur J Clin Nutr. 2007;61:1380-5.

13. PNAC en: https://www.minsal.cl/wpcontent/uploads/2015/09/2016.04.20Norma-T\%C3\%A9cnica-Prog.-
Alimentarios-aprobada-porJur\%C3\%ADdica.pdf

14. Voller SMB. Follow-Up Care for HighRisk Preterm Infants. Pediatr Ann. 2018;47:e142-e146.

15. Fenton TR, Nasser R, Eliasziw M, et al. Validating the weight gain of preterm infants between the reference growth curve of the fetus and the term infant. BMC Pediatrics 2013;13:92.

16. WHO Multicenter Growth Reference Study Group: WHO Child Growth Standards. Acta Pediatr Suppl. 2006;450:5-101.

17. Lafeber $\mathrm{H}$, van de Lagemaat $\mathrm{M}$, Rotteveel J, and van Weissenbruch M Timing of nutritional interventions in very-low-birth-weight infants: optimal neurodevelopment compared with the onset of the metabolic syndrome. Am J Clin Nutr 2013;98(suppl):556S-60S.

18. Suikkanen J, Matinolli HM, Eriksson JG, et al. Early postnatal nutrition after preterm birth and cardiometabolic risk factors in young adulthood. PLoS One. 2018;13:e0209404.

19. Pampanini V, Boiani A, De Marchis C, et al. Preterm infants with severe extrauterine growth retardation (EUGR) are at high risk of growth impairment during childhood. Eur J Pediatr. 2015;174:33-41.

20. Neubauer V, Fuchs T, Griesmaier E, et al. Comparing growth charts demonstrated significant deviations between the interpretation of postnatal growth patterns in very preterm infants. Acta Paediatr. 2016;105:268-73.

21. Fenton TR, Cormack B, Goldberg D, et al. "Extrauterine growth restriction" and "postnatal growth failure" are misnomers for preterm infants. J Perinatol. 2020;40:704-14.

22. Tuzun F, Yucesoy E, Baysal B, et al. Comparison of INTERGROWTH-21 and Fenton growth standards to assess size at birth and extrauterine growth in very preterm infants. J. Matern. Fetal Neonatal Med. 2018;31:2252-7.

23. Rochow N, Landau-Crangle E, So HY, et al. Z-score differences based on crosssectional growth charts do not reflect the growth rate of very low birth weight infants. PLoS One. 2019;14(5):e0216048.

24. Zozaya, Carlos. How Should We Define Postnatal Growth Restriction in Preterm Infants? Neonatology 2018;114:177-80.

25. Milad M, Novoen J, Fabres J, et al. Recomendación sobre curvas de crecimiento intrauterino Rev Chil Pediatr. 2010;81:264-74.

26. New global estimates on preterm birth published. World Health Organization. [cited 2019 May 29]. Available from: https://www.who.int/ reproductivehealth/global-estimates- preterm-birth/en/

27. Figueras-Aloy J, Palet-Trujols C, Matas-Barceló I, et al. Extrauterine growth restriction in very preterm infant: etiology, diagnosis, and 2-year follow-up. Eur J Pediatr. 2020;179:146979.

28. Avila-Alvarez A, Solar Bog A, BermúdezHormigoa C, et al. Restricción del crecimiento extrauterino en recién nacidos de menos de 1.500 gramos de peso al nacer. An Pediatr. (Barc) 2018;89:325-32.

29. Tabicas PA, Carvalho M, Carioca AC, et al. Variables associated with extrauterine growth restriction in very low birth weight infants. J Pediatr. (Rio J) 2014;90:22-7.

30. Asbury MR, Unger S, Kiss A, et al. Optimizing the growth of very-lowbirth-weight infants requires targeting both nutritional and nonnutritional modifiable factors specific to stage of hospitalization. Am J Clin Nutr. 2019;110(6):1384-94.

31. Goswami I, Rochow N, Fusch G, Liu K, Marrin ML, Heckmann M, Nelle M, Fusch C. Length Normalized Indices for Fat Mass and Fat-Free Mass in Preterm and Term Infants during the First Six Months of Life. Nutrients. $2016 ; 8: 417$.

32. Alur P. Sex Differences in Nutrition, Growth, and Metabolism in Preterm Infants. Front Pediatr. 2019;7:22.

33. Tottman AC, Bloomfield FH, Cormack BE, et al. Sex-specific relationships between early nutrition and neurodevelopment in preterm infants. Pediatr Res. 2020;87:872-8.

34. Teller IC, Embleton ND, Griffin IJ, et al. Post-discharge formula feeding in preterm infants: A systematic review mapping evidence about the role of macronutrient enrichment. Clin Nutr. 2016;35:791-801.

35. Cooke RJ, Griffin IJ, McCormick K, et al. Feeding preterm infants after hospital discharge: effect of dietary manipulation on nutrient intake and growth. Pediatr Res. 1998;43:355-60.

36. Bell KA, Matthews LG, Cherkerzian S, et al. Associations of Growth and Body Composition with Brain Size in Preterm Infants. J Pediatr. 2019;214:20-26.e2.

37. Watanabe $\mathrm{Y}$, Itabashi $\mathrm{K}$, Taki $\mathrm{M}$, et al. Body length and occipitofrontal circumference may be good indicators of neurodevelopment in very low birthweight infants - secondary publication. Acta Paediatr. 2018;107:975-80.

38. Gutbrod T, Wolke D, Soehne B, et al. Effects of gestation and birth weight on the growth and development of very low birthweight small for gestational age infants: a matched group comparison. Arch Dis Child Fetal Neonatal Ed. 2000;82:F208-14. 